Arq. Bras. Med. Vet. Zootec., v.69, n.5, p.1155-1162, 2017

\title{
Avaliação clínica de ovinos após a ingestão de elevada quantidade de manga
}

\author{
[Clinical evaluation of sheep after high ingestion of mango fruit] \\ G.W.N. Soares ${ }^{1}$, P.R. Lima ${ }^{1}$, A.S. Oliveira ${ }^{1}$, A.R.F. Lucena ${ }^{1}$, J.C. Silva Nascimento ${ }^{1}$, J.C. Matos ${ }^{1}$, \\ J.R. Amorim², D.R. Menezes ${ }^{3}$, A.C. Antonelli ${ }^{3 *}$ \\ ${ }^{1}$ Aluno de pós-graduação - Universidade Federal do Vale do São Francisco - Univasf - Petrolina, PE \\ ${ }^{2}$ Aluno de graduação - Universidade Federal do Vale do São Francisco - Univasf - Petrolina, PE \\ ${ }^{3}$ Universidade Federal do Vale do São Francisco - Univasf - Petrolina, PE
}

\begin{abstract}
RESUMO
Objetivou-se avaliar clinicamente os efeitos da administração intrarruminal de duas quantidades distintas de manga em ovinos. Foram utilizados sete ovinos machos, hígidos, que não receberam carboidratos não fibrosos por, pelo menos, seis meses previamente ao período experimental, quando se avaliou $\mathrm{pH}$ ruminal, total de protozoários no suco de rúmen, $\mathrm{pH}$ urinário, $\mathrm{pH}$ sanguíneo estimado e parâmetros vitais nos tempos zero, 12, 16, 20 e 24 após a administração da manga. Os sete ovinos foram distribuídos aleatoriamente em dois grupos e receberam $0,625 \%$ ou $1,875 \%$ da MS de manga/kg/PV (M1 e M2, respectivamente), distribuídos em delineamento cross-over, com uma parcela perdida, e 30 dias de washout. Os ovinos M2 apresentaram valores médios para $\mathrm{pH}$ ruminal significativamente inferiores a M1 a partir do tempo T12 (5,1 e 6,9, respectivamente), o que indica ocorrência de acidose ruminal. Os animais tratados com M1 não apresentaram alterações sistêmicas, ao passo que os ovinos tratados com M2 apresentaram acidose metabólica leve, detectada por meio do $\mathrm{pH}$ urinário ácido $(4,8)$. A ingestão apenas de manga in natura na quantidade de $1,875 \%$ da MS de manga/kg/PV mostrou-se capaz de provocar acidose ruminal em ovinos, levando-os a: ligeira depressão, aumento da frequência cardíaca, diminuição dos movimentos ruminais e diarreia em alguns casos.
\end{abstract}

Palavras-chave: acidose, rúmen, manga, pequenos ruminantes

\begin{abstract}
The aim of this study is to clinically evaluate the effects of intra ruminal administration of two different amounts of mango in sheep. The sample was of seven male healthy sheep, which did not receive nonfibrous carbohydrates for at least six months prior to the trial. Rumen fluid $\mathrm{pH}$, total of protozoa in the rumen fluid, urine $\mathrm{pH}$, estimated blood $\mathrm{pH}$, and vital parameters were evaluated at the following times: zero, 12, 16, 20 and 24 hours after administration of the mango. The seven sheep were randomly divided into two groups and received either $0.625 \%$ or $1.875 \%$ of the dry matter of the pulp and mango peel per $\mathrm{kg}$ body weight (M1 and M2, respectively), distributed in cross-over design with a lost portion and 30 days washout. Sheep treated with M2 showed significantly lower average values for ruminal $p H$ than the M1 since T12 (5.1 and 6.9, respectively), indicating the occurrence of ruminal acidosis. The animals treated with M1 showed no systemic changes, while the sheep treated with M2 had mild metabolic acidosis, detected through the lower urinary $\mathrm{pH}$ (4.8). The in natura mango ingestion in the amount of $1.875 \%$ of the dry matter of mango per $\mathrm{kg}$ of body weight proved to provoke rumen acidosis in sheep, leading these animals to: slight depression, rise in heart rate, diminished rumen movement, and diarrhea in some cases.
\end{abstract}

Keywords: acidosis, rumen, mango, small ruminants

Recebido em 10 de novembro de 2016

Aceito em 6 de janeiro de 2017

*Autor para correspondência (corresponding author)

E-mail: alexandre.antonelli@univasf.edu.br 


\section{INTRODUÇÃO}

A alimentação dos ruminantes é baseada em alimentos fibrosos, acompanhada de simbiose com microrganismos que digerem a fibra vegetal (Silva et al., 2014). O processo digestivo nos ruminantes acontece de forma dinâmica, envolvendo desde a entrada de alimentos no rúmen à saída de líquidos, partículas, microrganismos e resíduos não degradados para o omaso e o abomaso (Pereira et al., 2002).

O rúmen depende de materiais fibrosos que agem fisiologicamente regulando seu $\mathrm{pH}$ e influenciando na dinâmica do crescimento da população bacteriana. Desequilíbrios no $\mathrm{pH}$ do suco ruminal são produzidos com a ingestão de altas quantidades de carboidratos altamente fermentáveis sem a prévia adaptação, o que pode levar à ocorrência da acidose ruminal (Oliveira et al., 2016).

Casos de acidose láctica ruminal que ocorrem naturalmente estão associados à ingestão não intencional de grãos em grandes quantidades, com influência do seu tipo e do processamento, além de tubérculos ou frutas com altas quantidades de amido ou açúcares, soro de leite e outros, ou também ao aumento do consumo de rações concentradas, ricas em carboidratos de rápida fermentação visando ao maior desempenho produtivo do animal (Miranda Neto et al., 2005; Nagaraja e Lechtenberg, 2007).

Devido à restrição alimentar na região semiárida e aos longos períodos de seca, a busca por fontes alimentares alternativas na dieta de ruminantes é constante, e a utilização de frutas e/ou subprodutos do seu processamento mostra-se uma excelente alternativa (Batista e Souza, 2015). Nas regiões de fruticultura irrigada, o excedente da produção de frutas é empregado na alimentação animal (Oliveira et al., 2015a). Há relatos do aproveitamento de abacaxi, maracujá, melão, manga, caju, goiaba, cupuaçu, entre outras frutas, na alimentação de ruminantes (Giordani Júnior et al., 2014; Oliveira et al., 2015a).

A literatura é escassa de trabalhos sobre os efeitos da substituição de frutas e suas alterações no estado clínico dos animais. Dessa forma, objetivou-se avaliar as alterações clínicas que a ingestão de manga em excesso pode provocar em ovinos hígidos.

\section{MATERIAL E MÉTODOS}

O presente estudo foi aprovado pelo Comitê de Ética e Deontologia em Estudos e Pesquisas (Certificado $\mathrm{n}^{\mathrm{o}}$ 0001/021014-CEDEP/Ceua) da Universidade Federal do Vale do São Francisco.

O experimento foi desenvolvido no Setor de Caprino e Ovinocultura, Campus Ciências Agrárias da Universidade Federal do Vale do São Francisco (Univasf), Petrolina, Pernambuco, Brasil (9'32' 16.31” S; 40 56'00.27” O).

Inicialmente iriam ser utilizados oito animais, mas um deles veio a óbito no dia anterior ao início do experimento, e, dessa forma, foram utilizados apenas sete ovinos machos, SRD, com peso médio de $33,5 \mathrm{~kg}$ de peso vivo e um ano e três meses de idade. Os animais foram mantidos em baias coletivas, com água e sal mineral ad libitum, alimentados com dieta basal composta de volumoso fresco (capim-elefante) oferecido em quantidade suficiente para que houvesse aproximadamente $10 \%$ de sobra diária, sendo descartadas as sobras e ofertado capim novo a cada dia.

Os animais não receberam nenhuma fonte de alimento à base de carboidratos não estrutural por, pelo menos, seis meses antes do período experimental, para manter um ambiente não adaptado à digestão de carboidratos não estruturais. Eles foram identificados com placas fixadas em colar de metal circulado com mangueira de plástico e everminados com vermífugo de amplo aspecto à base de moxidectina 1\% (Cydectin ${ }^{\circledR}$ ) 30 dias antes do início de período experimental.

Os animais foram pesados após um período de jejum alimentar de 24 horas e de jejum hídrico de 12 horas, para calcular a quantidade de manga a ser fornecida a cada animal, com base no peso vivo deles.

A manga foi obtida de frutos maduros da variedade Palmer, da qual foram aproveitadas a polpa e a casca, que foram processadas em liquidificar industrial inox de alta rotação. A mistura de polpa e casca foi acondicionada em sacos plásticos e armazenada em refrigerador em 
temperatura inferior a $-20^{\circ} \mathrm{C}$ até o momento do uso. A manga processada apresentou $18^{\circ}$ Brix, acidez de $0,34 \%$ e matéria seca de $21,96 \%$.

Foi utilizado delineamento cross-over, em que os animais foram divididos aleatoriamente em dois grupos com uma parcela perdida em um dos grupos, e cada grupo recebeu um dos dois tratamentos. Após um período de 30 dias de washout, o outro tratamento foi aplicado. $\mathrm{Ou}$ seja, cada grupo recebeu os dois tratamentos experimentais uma única vez, com intervalo de 30 dias entre os tratamentos.

Os tratamentos experimentais consistiram no fornecimento único e súbito de polpa e casca de manga madura, conforme a seguir:

Tratamento 1 (M1) - 0,625\% de M.S. de manga/kg de P.V.

Tratamento 2 (M2) - 1,875\% de M.S. de manga/kg de P.V.

Assim, de forma hipotética, um ovino com $33,5 \mathrm{~kg}$ recebeu $953 \mathrm{~g}$ de manga no M1 e $2.860 \mathrm{~g}$ de manga no $\mathrm{M} 2$.

A manga foi fornecida utilizando-se uma sonda orogástrica acoplada em bomba para administração de fluidos (Drench Pump).

Foram realizados exames físicos e coletas de suco ruminal e urina nos momentos: zero, 12, 16, 20 e 24 horas após o fornecimento da manga.

O exame clínico foi realizado segundo Dirksen et al. (1993), sendo observada atitude, e os parâmetros avaliados foram: temperatura retal, frequência cardíaca e respiratória, motilidade ruminal e aspectos das fezes.

O suco de rúmen foi coletado por meio de sonda orogástrica e bomba de sucção a vácuo elétrica, sendo desprezados os primeiros $200 \mathrm{~mL}$ de suco de rúmen obtidos para evitar a contaminação com saliva (Dirksen et al., 1993). A urina foi coletada mediante a fixação de coletores de urina infantil no prepúcio dos animais e estimulada por massagem prepucial. $\mathrm{O} \mathrm{pH}$ ruminal e o da urina foram mensurados imediatamente após a coleta, utilizando-se um pHmetro digital portátil previamente calibrado. $\mathrm{O}$ pH sanguíneo estimado foi calculado baseado no $\mathrm{pH}$ urinário, empregando-se a fórmula determinada por Maruta et al. (2008) para ruminantes:

$[\mathrm{pH}$ sanguíneo estimado $=0,0619 \times \mathrm{pH}$ urinário $+6,8868](\mathrm{r}=0,75 ; \mathrm{p}<0,0001)$.

Para realização da contagem total de protozoários presentes no suco ruminal, foi realizada técnica descrita por Dehority (1977).

Os dados obtidos foram primeiramente analisados quanto a sua distribuição normal pela prova de Kolmogorov-Smirnov. Posteriormente, esses dados foram avaliados segundo testes estatísticos paramétricos, por meio de teste $\mathrm{F}$ (análise de variância), e, quando significativas, as médias foram confrontadas pelo teste de Tukey-Kramer. Foram consideradas significativas as diferenças cujo valor de "p" apresentou valores iguais ou inferiores a 0,05 $(\mathrm{P} \leq 0,05)$. Para realizar as análises estatísticas, foi utilizado o programa computadorizado GraphPad InStat ${ }^{\circledR}$.

\section{RESULTADOS E DISCUSSÃO}

Em todo o período experimental em que os ovinos receberam $\mathrm{M} 1$, não houve alteração significativa no $\mathrm{pH}$ ruminal, conforme evidenciado na Tab. 1. Já ao receberem o tratamento $\mathrm{M} 2$, houve queda significativa do $\mathrm{pH}$ ruminal, que atingiu seus valores médios mais baixos no tempo T12, com pequena elevação a partir de T20, mas ainda significativamente inferior ao tempo inicial. Quando foram comparados os tratamentos, a partir do tempo T12, o tratamento M2 apresentou valores médios para $\mathrm{pH}$ ruminal significativamente inferiores a M1, conforme Tab. 1.

No tempo T12, dois animais tratados com M2 apresentaram valores de $\mathrm{pH}$ ruminal inferiores a 5,0 (4,7 e 4,8), três animais apresentaram valor de 5,1, um animal com $\mathrm{pH}$ ruminal de 5,3 e outro com 5,6. Segundo Nagaraja e Titgemeyer (2007), animais com $\mathrm{pH}$ ruminal entre 5,1 e 5,6 apresentam quadro de acidose ruminal subaguda, e abaixo de 5,1 um quadro de acidose aguda. Dessa forma, todos os animais que simularam a ingestão de uma dieta exclusiva de manga in natura em grande quantidade (M2) apresentaram quadro de acidose ruminal subaguda ou aguda. 
Tabela 1. Valores médios e erro-padrão das médias dos parâmetros ruminais ( $\mathrm{pH}$ ruminal e total de protozoários), $\mathrm{pH}$ de urina e $\mathrm{pH}$ sanguíneo estimado (Maruta et al., 2008) de ovinos que receberam diferentes quantidades de manga

\begin{tabular}{lcccccc} 
& Trat. & \multicolumn{5}{c}{ Tempo } \\
\cline { 3 - 7 } & & 0 & 12 & 16 & 20 & 24 \\
\hline pH ruminal & M1 & $6,89 \pm 0,13$ & $6,90 \pm 0,10 \mathrm{~A}$ & $6,97 \pm 0,09 \mathrm{~A}$ & $7,06 \pm 0,09 \mathrm{~A}$ & $7,17 \pm 0,09 \mathrm{~A}$ \\
& M2 & $6,84 \pm 0,09 \mathrm{a}$ & $5,10 \pm 0,11 \mathrm{cB}$ & $5,43 \pm 0,15 \mathrm{bcB}$ & $5,91 \pm 0,16 \mathrm{bB}$ & $6,14 \pm 0,30 \mathrm{bB}$ \\
Total de protoz. & M1 & $21,1 \pm 2,9$ & $16,1 \pm 2,7$ & $18,6 \pm 2,9 \mathrm{~A}$ & $14,3 \pm 3,0$ & $17,1 \pm 2,8$ \\
(X 104/mL) & M2 & $20,6 \pm 5,5 \mathrm{a}$ & $12,5 \pm 4,6 \mathrm{ab}$ & $7,4 \pm 0,9 \mathrm{bB}$ & $8,9 \pm 2,5 \mathrm{~b}$ & $10,6 \pm 2,0 \mathrm{ab}$ \\
& & & & & & \\
pH urinário & M1 & $6,7 \pm 0,5$ & $6,6 \pm 0,4$ & $6,9 \pm 0,4 \mathrm{~A}$ & $6,9 \pm 0,4 \mathrm{~A}$ & $6,3 \pm 0,4 \mathrm{~A}$ \\
& M2 & $7,2 \pm 0,4 \mathrm{a}$ & $6,0 \pm 0,4 \mathrm{~b}$ & $5,0 \pm 0,1 \mathrm{bcB}$ & $4,8 \pm 0,1 \mathrm{cB}$ & $5,2 \pm 0,2 \mathrm{bcB}$ \\
pH sanguíneo & & & & & \\
estimado & M1 & $7,30 \pm 0,03$ & $7,30 \pm 0,02$ & $7,32 \pm 0,02 \mathrm{~A}$ & $7,32 \pm 0,02 \mathrm{~A}$ & $7,28 \pm 0,02 \mathrm{~A}$ \\
\hline & M2 & $7,33 \pm 0,03 \mathrm{a}$ & $7,26 \pm 0,03 \mathrm{~b}$ & $7,19 \pm 0,01 \mathrm{bB}$ & $7,19 \pm 0,01 \mathrm{bB}$ & $7,21 \pm 0,01 \mathrm{bB}$ \\
\hline
\end{tabular}

Nota: Letras minúsculas distintas nas linhas indicam diferenças significativas entre os tempos. Letras maiúsculas distintas nas colunas indicam diferenças significativas entre os tratamentos $(\mathrm{P}<0,05)$.

Era esperado que o fornecimento exclusivo de manga na dieta de ovinos resultasse em quadro de acidose ruminal, pois Oliveira et al. (2015a) atingiram o mesmo objetivo ao fornecerem melão com Brix inferior $\left(12^{\circ} \mathrm{Bx}\right)$ ao da manga ofertada neste experimento $\left(18^{\circ} \mathrm{Bx}\right)$. Como o Brix estima com precisão o percentual do teor de sólidos solúveis totais presentes na manga processada, neste caso constituído, em sua maioria, por sacarose (até 76\%), além de frutose e glicose, pode-se considerar que $18 \%$ da manga madura eram compostos por açúcares (Bernardes-Silva et al., 2003; Official..., 2012). Assim, pode-se afirmar que, na situação hipotética do ovino com $33,5 \mathrm{~kg}$, foram fornecidos $171 \mathrm{~g}$ de açúcares no M1 e $514 \mathrm{~g}$ de açúcares no M2, perfazendo $5,1 \mathrm{~g} / \mathrm{kg}$ PV e $15,3 \mathrm{~g} / \mathrm{kg}$ PV, respectivamente. Reis (2011) obteve êxito ao induzir acidose láctica ruminal aguda em todos os ovinos, ao administrar $15 \mathrm{~g} / \mathrm{kg}$ PV de sacarose intrarruminalmente, valor semelhante ao utilizado no presente trabalho.

Foi verificado que todos os animais, quando receberam o M2, apresentaram uma ligeira depressão e perdiam o interesse por alimento, enquanto todos os animais, quando receberam M1, procuravam alimento no cocho desde o momento $12 \mathrm{~h}$. Durante o processo de instalação da acidose láctica ruminal, ocorre uma grande produção de ácido láctico dentro do rúmen, o que eleva, de forma considerável, a osmolaridade ruminal. Huber (1971) verificou um aumento da osmolaridade ruminal em ovinos de 255 para $402 \mathrm{mOsm} / \mathrm{L}$. Variações na osmolaridade ruminal são percebidas pelas paredes de rúmen e retículo, e valores acima de $300 \mathrm{mOsm} / \mathrm{L}$ são capazes de inibir a ingestão de alimento, os quais, ao ultrapassarem $350 \mathrm{mOsm} / \mathrm{L}$, inibem a digestão bacteriana de amido e fibras (Carter e Grovum, 1990).

Também foi verificado que dois animais submetidos a M2 $(28,5 \%)$ apresentaram diarreia durante o período experimental de 24 horas. Esse valor é superior ao encontrado por Reis (2011), que detectou diarreia em $16,7 \%$ dos animais com acidose ruminal. A presença da diarreia pode ser explicada também pelo aumento da osmolaridade do conteúdo do trato gastrointestinal, o que provoca uma diarreia osmótica ao atrair grande quantidade de líquido para a luz intestinal na tentativa de manter o equilíbrio osmótico. Apesar da diarreia, a frequência e o volume de defecação não sofrem grandes alterações (Maruta e Ortolani, 2002; Nagaraja e Lechtenberg, 2007).

Em relação à população total de protozoários, não foram encontradas diferenças significativas no decorrer do tempo para os animais que receberam M1, entretanto ocorreu uma diminuição significativa no tempo T16, que se manteve até o T20 (Tab. 1). Nagaraja e Titgemeyer (2007) verificaram que há uma correlação negativa entre o total de protozoários no líquido ruminal e a ocorrência de acidose ruminal. A diminuição apenas após 16 horas da indução contraria os achados de Miranda Neto et al. (2011), que verificaram essa diminuição ocorrendo a partir da quarta hora pós-indução de acidose láctica em caprinos. Esse fato fica mais evidente ao se comparar o total de protozoários 
no tempo T16, pois o tratamento M2 provocou uma diminuição significativa em relação ao tratamento M1 (Tab. 1).

A presença de protozoários é fundamental para reduzir a queda no $\mathrm{pH}$ ruminal e evitar ou atenuar acidose ruminal, pois protozoários digerem grânulos de amido mais lentamente que as bactérias, o que diminui seu efeito depressor no pH (Kozloski, 2002). Entretanto, quando há redução no $\mathrm{pH}$ ruminal, há uma redução concomitante na densidade de protozoários ruminais (Bürger et al., 2000).

Os ácidos graxos voláteis e lactato presentes no suco ruminal são absorvidos pelo mesmo mecanismo, apesar de serem utilizados de forma diferente pelas células (Graham et al., 2007). Em condições fisiológicas normais, os rins e o fígado são responsáveis por metabolizar e excretar o lactato (Allen e Holm, 2008). Mas quando este é absorvido em excesso, nos casos de acidose láctica ruminal, ele promove uma queda no $\mathrm{pH}$ sanguíneo, o que resulta em uma acidose metabólica (Maruta et al., 2008).

Um dos principais mecanismos do organismo para compensar a acidose metabólica é a excreção de íons $\mathrm{H}^{+}$via renal; nos casos de acidose láctica ruminal, há uma expressiva queda do pH urinário (Maruta et al., 2008), além de refletir o que ocorre em relação ao $\mathrm{pH}$ sanguíneo (Carlson e Bruss, 2008). O mecanismo compensatório da acidose metabólica é perceptível ao se verificar que o tratamento M2 resultou em uma diminuição progressiva significativa do $\mathrm{pH}$ urinário já a partir do T12 e atingiu seu valor médio mais baixo no tempo T20, enquanto o tratamento M1 não alterou, de forma significativa, o $\mathrm{pH}$ urinário (Tab. 1). Também foi determinado que, a partir do tempo T16 até o final (T24), os valores para $\mathrm{pH}$ urinário foram significativamente menores para $\mathrm{M} 2 \mathrm{em}$ relação ao M1.

Maruta et al. (2008) desenvolveram fórmula com base na determinação do $\mathrm{pH}$ urinário para estimar o $\mathrm{pH}$ sanguíneo e o status acidobásico em ruminantes com quadro de acidose ruminal, com a finalidade de ser um método prático, rápido, barato e preciso a campo para calcular a necessidade de bicarbonato na correção da acidose metabólica. Ao se aplicar essa fórmula nos resultados de $\mathrm{pH}$ urinário, verificou-se que o $\mathrm{pH}$ sanguíneo dos ovinos tratados com M2 diminuiu significativamente a partir de T12 e, a partir de T16, foi significativamente inferior aos valores de $\mathrm{pH}$ sanguíneo dos animais tratados com M1 (Tab. 1).

A estimativa do $\mathrm{pH}$ sanguíneo em casos em que não é possível determiná-lo por meio de aparelhos de hemogasometria é fundamental, pois pode-se instituir a correção da acidose metabólica mediante a administração de solução de bicarbonato intravenoso (Ortolani, 2003). O limite mínimo para $\mathrm{pH}$ sanguíneo de ovinos adultos criados em condições brasileiras é 7,28, o que leva a interpretar que os ovinos tratados com M2 apresentaram acidose leve a partir de T12 e atingiram acidose metabólica moderada a partir de T16, com o valor médio de 7,19.

Na avaliação dos parâmetros vitais, constatou-se que, quando os animais receberam M1, a frequência cardíaca manteve-se dentro da normalidade, sem diferir significativamente do T0, conforme pode-se verificar na Tab. 2. Já quando os animais receberam M2, a frequência cardíaca apresentou seus valores significativamente mais elevados no $\mathrm{T} 12$, mantendo-se mais elevada que o T0 também nos tempos T16 e T20 e retornando a valores similares aos basais apenas no final do período experimental (T24).

Quando são comparados os resultados entre os tratamentos, tanto no T12 como no T16, os valores apresentados pelos animais ao receberam M2 foram significativamente superiores do que quando receberam M1, conforme Tab. 2. Segundo Ortolani et al. (2010), casos clássicos de acidose láctica ruminal levam ao aumento da frequência cardíaca, devido ao aumento do grau de desidratação. Entretanto, Oliveira et al. (2015b) verificaram a ocorrência de taquicardia sem que os animais apresentassem o quadro de desidratação em casos de acidose láctica ruminal e atribuíram o aumento na frequência cardíaca ao aumento do volume abdominal, fato que também deve ter ocorrido no presente trabalho. 
Tabela 2. Valores médios e erro-padrão das médias dos parâmetros vitais (frequência cardíaca, frequência respiratória, movimentos ruminais e temperatura) de ovinos que receberam diferentes quantidades de manga

\begin{tabular}{|c|c|c|c|c|c|c|}
\hline & \multirow{2}{*}{ Trat. } & \multicolumn{5}{|c|}{ Tempo } \\
\hline & & 0 & 12 & 16 & 20 & 24 \\
\hline Frequência cardíaca & M1 & $68,6 \pm 2,5$ & $82,3 \pm 7,5 \mathrm{~B}$ & $73,1 \pm 3,0 \mathrm{~B}$ & $74,3 \pm 6,8$ & $73,1 \pm 4,3$ \\
\hline$(\mathrm{bpm})$ & M2 & $70,3 \pm 2,6 \mathrm{c}$ & $109,1 \pm 5,5 \mathrm{aA}$ & $86,9 \pm 4,8 \mathrm{bA}$ & $88,0 \pm 4,1 \mathrm{~b}$ & $80,6 \pm 5,1 \mathrm{bc}$ \\
\hline Frequência & M1 & $24,0 \pm 2,1$ & $24,6 \pm 2,2$ & $25,7 \pm 1,7$ & $24,6 \pm 2,0$ & $23,4 \pm 2,8$ \\
\hline respiratória (mpm) & M2 & $25,1 \pm 1,1$ & $24,6 \pm 1,0$ & $22,9 \pm 1,9$ & $26,3 \pm 2,3$ & $23,4 \pm 1,8$ \\
\hline Movimentos ruminais & M1 & $2,1 \pm 0,2 \mathrm{a}$ & $1,4 \pm 0,2 b$ & $0,8 \pm 0,1 \mathrm{~b}$ & $0,9 \pm 0,2 \mathrm{bA}$ & $0,9 \pm 0,3 b$ \\
\hline$(\mathrm{mov} / 2 ")$ & M2 & $1,7 \pm 0,2 \mathrm{a}$ & $1,0 \pm 0,3 b$ & $0,4 \pm 0,2 \mathrm{bc}$ & $0,1 \pm 0,1 \mathrm{cB}$ & $0,4 \pm 0,3 \mathrm{bc}$ \\
\hline Temperatura retal $\left(^{\circ}\right.$ & M1 & $38,6 \pm 0,3 \mathrm{ab}$ & $38,0 \pm 0,3 \mathrm{bB}$ & $38,4 \pm 0,2 \mathrm{bB}$ & $39,3 \pm 0,3 \mathrm{a}$ & $39,3 \pm 0,2 \mathrm{a}$ \\
\hline C) & M2 & $39,1 \pm 0,1 \mathrm{ab}$ & $38,9 \pm 0,3 \mathrm{bA}$ & $39,1 \pm 0,2 \mathrm{abA}$ & $39,4 \pm 0,1 \mathrm{ab}$ & $39,5 \pm 0,1 \mathrm{a}$ \\
\hline
\end{tabular}

Nota: Letras minúsculas distintas nas linhas indicam diferenças significativas entre os tempos. Letras maiúsculas distintas nas colunas indicam diferenças significativas entre os tratamentos $(\mathrm{P}<0,05)$.

Em relação à frequência respiratória, não houve variação entre os tratamentos, tampouco no decorrer do tempo experimental, conforme mostra a Tab. 2. A frequência respiratória, durante todo o período experimental, para ambos os tratamentos, encontrou-se no patamar considerado como aumentado no trabalho de Oliveira et al. (2015b), o que pode ser explicado pelo fato de os animais deste experimento estarem em um ambiente com temperatura elevada, o que naturalmente resulta em taquipneia (Andrade et al., 2007). A dissipação do calor por evaporação por meio da respiração é um dos mecanismos mais importantes para dissipar calor para raças adaptadas ao estresse térmico (Medeiros et al., 2008). A temperatura diária nos dias em que ocorreram os ensaios variou de $28^{\circ} \mathrm{C}$ pela manhã a $38^{\circ} \mathrm{C}$ à tarde, o que explica a variação na temperatura retal em ambos os tratamentos no decorrer do tempo.

Outro fator que colabora para a dissipação de calor é a perda de calor corpóreo para a água ingerida (Yousef, 1985), o que pode explicar a razão de dos ovinos, ao serem submetidos ao tratamento M2, apresentarem temperatura retal superior ao M1 nos tempos T12 e T16 (Tab. 2), pois, por estarem ligeiramente deprimidos, não tinham interesse também na ingestão de água, o que supostamente prejudicou a dissipação de calor.

Houve uma diminuição significativa nos movimentos ruminais quando os animais receberam o M1 (Tab. 2), mas esse fato pode-se atribuir ao jejum a que esses animais estavam submetidos, pois nenhum deles apresentou atonia ruminal, característica de animais com acidose láctica ruminal (Ortolani et al., 2010). O jejum promove diminuição do conteúdo ruminal e consequente diminuição de estímulos excitatórios nos receptores de baixa tensão limiar no retículo, além da não ativação dos receptores bucais durante a mastigação (Reece, 2007). Já em relação ao período em que os ovinos receberam o $\mathrm{M} 2$, seis animais $(85,7 \%)$ apresentaram atonia ruminal desde o tempo T12, e, ao final do T24, quatro animais $(57,1 \%)$ ainda apresentavam atonia ruminal. Oliveira et al. (2015b) já relataram que o $\mathrm{pH}$ ruminal baixo inibe a motilidade ruminal, e, quando o $\mathrm{pH}$ é inferior a 5,0, ocorre atonia. Neste trabalho, verificou-se que não apenas os animais com $\mathrm{pH}$ ruminal abaixo de 5,0 apresentaram atonia ruminal, mas também animais com $\mathrm{pH}$ acima de 5,0 , com um indivíduo apresentando $\mathrm{pH}$ ruminal de 5,7. O aumento do volume abdominal colabora com o desencadeamento do quadro de atonia ruminal, pois há um aumento de estímulos inibitórios receptores de alta tensão limiar no retículo e no saco cranial do rúmen (Reece, 2007).

Ao final das 24 horas de monitoramento experimental, a dieta à base de capim-elefante picado foi reintroduzida. Todos os animais, quando receberam M1, alimentaram-se normalmente, e quando tratados com M2, ocorreu um retorno gradativo do apetite. Todos os ovinos que apresentaram acidose ruminal recuperaram-se espontaneamente, não sendo realizado nenhum tipo de tratamento de suporte. 


\section{CONCLUSÕES}

A administração de $1,875 \%$ da matéria seca de manga por $\mathrm{kg}$ de peso vivo a ovinos não adaptados ao consumo elevado de carboidratos pode resultar em acidose ruminal subaguda ou aguda de forma transitória, manifestada na queda do $\mathrm{pH}$ ruminal, atonia ruminal, levando ao desenvolvimento de uma acidose metabólica leve a moderada, indicada pela queda no $\mathrm{pH}$ urinário.

\section{REFERÊNCIAS}

ALLEN, S.E.; HOLM, J.L. Lactate: physiology and clinical utility. J. Vet. Emerg. Crit. Car., v.18, p.123-132, 2008.

ANDRADE, I.S.; SOUZA, B.B.; PEREIRA FILHO, J.M.; SILVA, A.M.A. Parâmetros fisiológicos e desempenho de ovinos Santa Inês submetidos a diferentes tipos de sombreamento e a suplementação em pastejo. Cienc. Agrotec., v.31, p.540-547, 2007.

BATISTA, N.L.; SOUZA, B.B. Caprinovinocultura no semiárido brasileiro - fatores limitantes e ações de mitigação. Agropecu. Cient. Semiárido, v.11, p.1-9, 2015.

BERNARDES-SILVA, A.P.F.; LAJOLO, F.M.; CORDENUNSI, B.R. Evolução dos teores de amido e açúcares solúveis durante o desenvolvimento e amadurecimento de diferentes cultivares de manga. Cienc. Tecnol. Aliment., v.23, p.116-120, 2003.

BÜRGER, P.J.; PEREIRA, J.C.; VALADARES FILHO, S.C. et al. Fermentação ruminal e eficiência microbiana em bezerros holandeses alimentados com dietas contendo diferentes níveis de concentrado. Rev. Bras. Zootec., v.26, p.215-224, 2000.

CARLSON G.P.; BRUSS, M.L. Fluid, electrolyte, and acid-base balance. In: KANEKO, J.J.; HARVEY, J.W; BRUSS, M.L. (Eds.) Clinical biochemistry of domestic animals. 6.ed. San Diego: Academic Press, 2008. p.529-559.

CARTER, R.R.; GROVUM, W.L. A review of the physiological significance of hypertonic body fluids on feed intake and ruminal function: salivation, motility and microbes. J. Anim. Sci., v.68, p.2811-2832,1990.
DEHORITY, B.A. Classification and morphology of rumen protozoa. Wooster: Ohio Agricultural Research and Development Center, 1977. 82p.

DIRKSEN, G.; GRÜNDER, D.; STÖBER, M. Rosenberger: exame clínico dos bovinos. 3.ed. Rio de Janeiro: Guanabara Koogan, 1993. 448p.

GIORDANI JÚNIOR, R.; CAVALI, J.; PORTO, M.O. et al. Resíduos agroindustriais e alimentação de ruminantes. Rev. Bras. Cienc. Amazônia, v.3, p.93-104, 2014.

GRAHAM, C.; GATHERAR, I.; HASLAM, I. et al. Expression and localization of monocarboxylate transporters and sodium/proton exchangers in bovine rumen epithelium. Am. J. Physiol. Regul. Integr., v.292, p.R997-R1007, 2007.

HUBER, T.L. Effect of acute indigestion on the compartmental water volumes and osmolality in sheep. Am. J. Vet. Res., v.32, p.887-890, 1971.

KOZLOSKI, G.V. Bioquímica dos ruminantes. Santa Maria: UFSM, 2002. 140p.

MARUTA, C.A.; LEAL, M.L.R.; MENDES NETTO, D. et al. The measurement of urine $\mathrm{pH}$ to predict the amount of buffer used in the treatment of acute rumen lactic acidosis in cattle. Cienc. Rural, v.38, p.712-722, 2008.

MARUTA, C.A.; ORTOLANI, E.L. Susceptibilidade de bovinos das raças Jersey e Gir à acidose láctica ruminal: I - variáveis ruminais e fecais. Cienc. Rural, v.32, p.55-59, 2002.

MEDEIROS, L.F.D.; VIEIRA, D.H.; OLIVEIRA, C.A. et al. Reações fisiológicas de caprinos das raças Anglo-nubiana e Saanen mantidos à sombra, ao sol e em ambiente parcialmente sombreado. Bol. Ind. Anim., v.65, p.7-14, 2008.

MIRANDA NETO, E.G.; AFONSO, J.A.B.; MENDONÇA, C.L.; ALMEIDA, M.Z.P.R.B. Estudo clínico e características do suco ruminal de caprinos com acidose láctica induzida experimentalmente. Pesqui. Vet. Bras., v.25, p.73-78, 2005.

MIRANDA NETO, E.G.; SILVA, S.T.G.; MENDONÇA, C.L. et al. Aspectos clínicos e a bioquímica ruminal de caprinos submetidos à acidose láctica experimental e suplementados ou não com monensina sódica. Pesqui. Vet. Bras., v.31, p.416-424, 2011. 
NAGARAJA， T.G.; LECHTENBERG， K.F. Acidosis in feedlot cattle. Vet. Clin. N. Am. Food A., v.23, p.333-350, 2007.

NAGARAJA, T.G.; TITGEMEYER, E.C. Ruminal acidosis in beef cattle: the current microbiological and nutritional outlook. J. Dairy Sci., v.90, p.E17-E38, 2007.

OFFICIAL Methods of Analysis of the Association of Official Analytical Chemists. 19.ed. Washington: AOAC, 2012. 3000p.

OLIVEIRA, F.L.C.; BARRETO JÚNIOR, R.A.; MINERVINO, A.H.H. et al. Avaliação hemogasométrica, bioquímica e hematológica de ovinos suplementados com melão. Arq. Bras. Med. Vet. Zootec., v.67, p.1272-1278, 2015a.

OLIVEIRA, F.L.C.; BARRETO JÚNIOR, R.A.; MINERVINO, A.H.H. et al. Clinical evaluation of unadapted sheep submited to sudden intake of melon with high levels of sugar. Semin. Cienc. Agrar., v.36, p.3721-3730, 2015 b.

OLIVEIRA, F.L.C.; BARRETO JÚNIOR, R.A.; MINERVINO, A.H.H. et al. Effects of sudden melon intake on ruminal parameters of non-adapted sheep. Pesqui. Vet. Bras., v.36, p.378-382, 2016.

ORTOLANI, E.L. Diagnóstico e tratamento de alterações ácido-básicas em ruminantes. In: SIMPÓSIO DE PATOLOGIA CLÍNICA VETERINÁRIA DA REGIÃO SUL DO BRASIL, 1., 2003, Porto Alegre. Anais... Porto Alegre: UFRGS, 2003. p.17-29.
ORTOLANI, E.L.; MARUTA, C.A.; MINERVINO, A.H.H. Quadro clínico de zebuínos e taurinos submetidos à acidose láctica ruminal aguda. Braz. J. Vet. Res. Anim. Sci., v.47, p.253-261, 2010.

PEREIRA, J.C.; ALMEIDA, M.S.; CECON, P.R.; QUEIROZ, A.C. Dinâmica da degradação ruminal por novilhos mantidos em pastagem natural, em diferentes épocas do ano. Rev. Bras. Zootec., v.31, p.740-748, 2002.

REECE, W.O. Dukes - fisiologia dos animais domésticos. 12.ed. Rio de Janeiro: Guanabara Koogan, 2007. 954p.

REIS, L.F. Estudo comparativo do uso de probiótico e monensina na prevenção $e$ tratamento da acidose láctica ruminal. 2011. 115f. Dissertação (Mestrado em Clínica Veterinária) - Faculdade de Medicina Veterinária e Zootecnia, Universidade de São Paulo, SP.

SILVA, K.L.; DUARTE, E.R.; FREITAS, C.E.S. et al. Protozoários ruminais de novilhos de corte criados em pastagem tropical durante o período seco. Cienc. Anim., v.15, p.259-265, 2014.

YOUSEF, M.K. Stress physiology in livestock. Boca Raton: CRC Press. 1985. v.2, 272p. 